
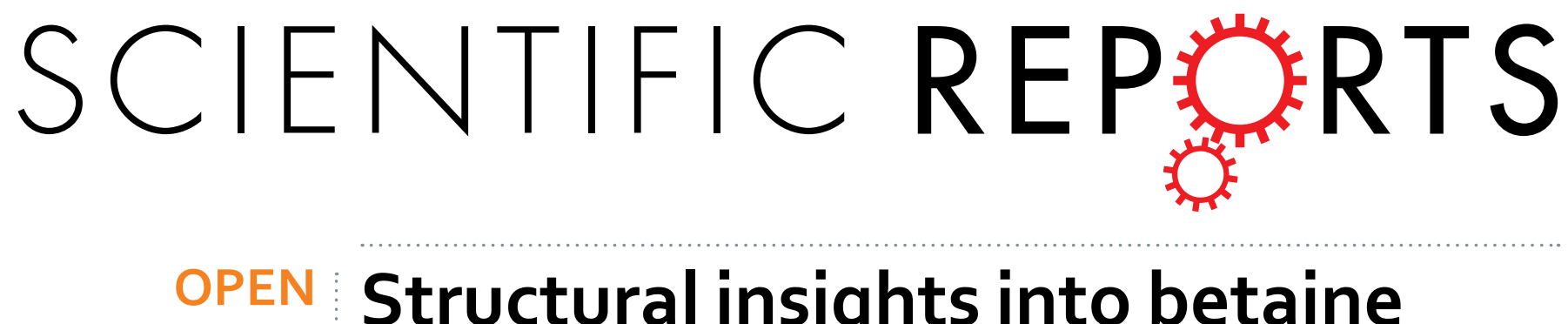

Structural insights into betaine aldehyde dehydrogenase (BADH2) from Oryza sativa explored by

Received: 30 April 2018

Accepted: 10 August 2018

Published online: 27 August 2018

\section{modeling and simulations}

Apisara Baicharoen ${ }^{1}$, Ranjit Vijayan² \& Prapasiri Pongprayoon $\mathbb{D I}^{1,3,4}$

Betaine aldehyde dehydrogenase 2 (BADH2) plays a key role in the accumulation of 2-acetyl-1-pyrroline (2AP), a fragrant compound in rice (Oryza sativa). BADH2 catalyses the oxidation of aminoaldehydes to carboxylic acids. An inactive BADH2 is known to promote fragrance in rice. The 3D structure and atomic level protein-ligand interactions are currently unknown. Here, the 3D dimeric structure of BADH2 was modeled using homology modeling. Furthermore, two $0.5 \mu \mathrm{s} \mathrm{simulations} \mathrm{were} \mathrm{performed} \mathrm{to} \mathrm{explore}$ the nature of BADH2 dimer structurally and dynamically. Each monomer comprises of 3 domains (substrate-binding, $\mathrm{NAD}^{+}$-binding, and oligomerization domains). The $\mathrm{NAD}^{+}$-binding domain is the most mobile. A scissor-like motion was observed between the monomers. Inside the binding pocket, N162 and E260 are tethered by strong hydrogen bonds to residues in close proximity. In contrast, the catalytic $\mathrm{C} 294$ is very mobile and interacts occasionally with N162. The flexibility of the nucleophilic C294 could facilitate the attack of free carbonyl on an aldehyde substrate. Key inter-subunit salt bridges contributing to dimerization were also identified. E487, D491, E492, K498, and K502 were found to form strong salt bridges with charged residues on the adjacent monomer. Specifically, the nearly permanent R430-E487 hydrogen bond ( $>90 \%$ ) highlights its key role in dimer association. Structural and dynamic insights of BADH2 obtained here could play a role in the improvement of rice fragrance, which could lead to an enhancement in rice quality and market price.

Rice (Oryza sativa) has many flavours and textures that impact on its quality. Fragrance is a key factor that determines rice quality. Fragrant rice is gaining worldwide popularity among consumers. Its market price is much higher than common varieties of nonfragrant rice. Due to an increasing demand on fragrant rice worldwide, many studies have focussed on increasing yield of fragrant rice and retaining their fragrance ${ }^{1,2}$. 2-acetyl-1-pyrroline (2AP) has been found to be the most potent flavour compound that gives unique fragrance to jasmine and basmati rice ${ }^{3}$. Thus, the level of $2 \mathrm{AP}$ in rice serves as a key factor in determining the market price of fragrant rice.

Betaine aldehyde dehydrogenase $2(\mathrm{BADH} 2)$ has been reported to play a role in the level of $2 \mathrm{AP}$ in rice ${ }^{4,5}$. The loss of $\mathrm{BADH} 2$ function accounts for the accumulation of $2 \mathrm{AP}$ resulting in an increase in rice fragrance ${ }^{5}$. BADH2 belongs to the aldehyde dehydrogenase $(\mathrm{ALDH})$ family whose members include $\mathrm{NAD}(\mathrm{P})^{+}$-dependent enzymes catalysing the oxidation of many intermediate aldehydes to their corresponding carboxylic acids ${ }^{6}$. BADH2 oxidizes a broad range of aminoaldehydes (e.g. $\gamma$-aminobutyraldehyde (GAB-ald), 3-aminopropionaldehyde (AP-ald), and 4-N-trimethylaminobutyraldehyde (TMAB-ald)) in addition to its natural substrate, betaine aldehyde (Bet-ald ${ }^{7-9}$. Furthermore, previous studies demonstrated that BADH2 catalyses the oxidation of GAB-ald more efficiently than Bet-ald ${ }^{4,7}$. Generally, BADH2 converts GAB-ald into GABA, but the absence or nonfunction of $\mathrm{BADH} 2$ results in the accumulation of GAB-ald which is subsequently converted to $2 \mathrm{AP}$.

\footnotetext{
${ }^{1}$ Department of Chemistry, Faculty of Science, Kasetsart University, Chatuchak, Bangkok, 10900, Thailand. ${ }^{2}$ Department of Biology, College of Science, United Arab Emirates University, PO Box, 15551, Al Ain, Abu Dhabi, United Arab Emirates. ${ }^{3}$ Center for Advanced Studies in Nanotechnology for Chemical, Food and Agricultural Industries, KU Institute for Advanced Studies, Kasetsart University, Bangkok, 10900, Thailand. ${ }^{4}$ Computational Biomodelling Laboratory for Agricultural Science and Technology (CBLAST), Kasetsart University, Bangkok, 10900, Thailand. Correspondence and requests for materials should be addressed to R.V. (email: ranjit.v@uaeu.ac.ae) or P.P. (email: fsciprpo@ku.ac.th)
} 

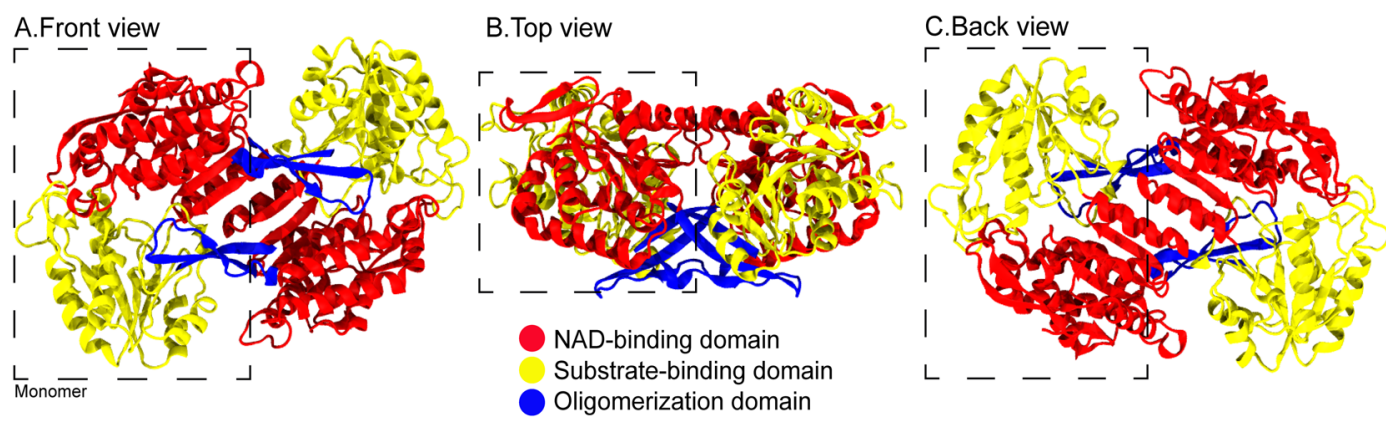

Figure 1. (A-C) Cartoon views of dimeric BADH2 with 3 domains (NAD ${ }^{+}$-binding domain in red, substratebinding domain in yellow, and oligomerization domain in blue). The dashed line represents a monomer.

Currently, two crystal structures of AMADH from Pisum sativum are available ${ }^{10}$. Both are dimeric. Each AMADH unit contains a NAD ${ }^{+}$-binding domain, an oligomerization domain, and a substrate-binding or catalytic domain (Fig. 1). In the catalytic domain, the catalytic triad (N162, E260, and C294) is conserved in many species. Two BADH homologs, BADH1 and BADH2, from Oryza sativa share $~ 75 \%$ sequence similarity between the two and $\sim 76 \%$ with AMADH. BADH1 was found to be mainly responsible for abiotic stress tolerance, while $\mathrm{BADH} 2$ contributes to rice aroma ${ }^{4,8,11}$. The level of BADH2 catalytic activity directly impacts the $2 \mathrm{AP}^{\mathrm{B}}$ evel $^{4,8}$. Since the function of $\mathrm{BADH} 2$ is attributed to the aroma of rice, many studies have been devoted to understanding the nature of rice $\mathrm{BADH} 2^{11-18}$. The ability to enhance rice aroma via controlling BADH2 activity can result in better rice quality and, importantly, higher market price.

$\mathrm{BADH} 2$ is found to be a dimer in plant ${ }^{10}$. For rice, each subunit (503 amino acids each) contains 3 domains ( $\mathrm{NAD}^{+}$-binding, substrate-binding, and oligomerization domains) (Fig. 1). The long oligomerization domain latches two units together (coloured blue in Fig. 1). Previous simulation and mutagenesis studies have identified a few key residues (N162, Y163, M167, W170, E260, W288, S295, W420, C453, and W459) that are crucial for substrate binding and recognition ${ }^{16-18}$. Mainly, N162, E260, C294 were found to play a role in catalytic activity (Fig. 1C). C294 and E260 were involved in a key step of hemithioacetal-enzyme formation, while N162 helps stabilize an intermediate ${ }^{16-18}$. However, no clear atomic level evidence is available. Earlier mutagenesis work reported some key residues for $\mathrm{BADH} 2$ function and the dynamics of these residues was observed in a presence of bound substrate using molecular dynamics (MD) simulations ${ }^{16-18}$. However, such short simulations may not be sufficient to explore most of the dynamic and structural properties of BADH2. Thus, in this work, we employed $0.5 \mu \mathrm{s}$ MD simulations to primarily observe the nature of native BADH2 in solution. Key structural and dynamic properties of $\mathrm{BADH} 2$ were also investigated. Structural insights of $\mathrm{BADH} 2$ obtained here will serve as a first step to understand the mechanism of fragrance production in rice which will be useful for future improvement to the fragrance of rice.

\section{Results}

ClustalW webserver (https://embnet.vital-it.ch/software/ClustalW.html) was used to align and calculate the sequence similarity between $\mathrm{BADH} 1$ and $\mathrm{BADH} 2$ from rice, and $\mathrm{AMADH}$ from P. sativum ${ }^{19}$. Both BADH1 and $\mathrm{BADH} 2$ share $75.94 \%$ sequence similarity, while $76.54 \%$ sequence similarity was found between BADH2 and AMADH. Almost all the key residues in the substrate- and NAD ${ }^{+}$-binding domains, such as N162, E260, and C294, are conserved (Fig. S2 in Supplementary Information). The crystal structure of AMADH was used as the template to construct a $3 \mathrm{D}$ model of $\mathrm{BADH} 2$ using homology modelling. To explore structural changes and flexibility, we computed the root mean-square deviations (RMSDs) and fluctuations (RMSFs) of the three-dimensional BADH2 model in simulations. RMSD was calculated by comparing the movement of atoms with initial coordinates at $t=0$. In general, $C \alpha$ RMSD (Fig. 2A) indicated that the core protein structure became stable after $10 \mathrm{~ns}$. Considering the individual domains, oligomerization and $\mathrm{NAD}^{+}$-binding domains are divided into 2 regions (residues 9-124 and 152-262 for $\mathrm{NAD}^{+}$-binding domain and residues 129-151 and 480-486 for oligomerization domain shown in Fig. 2E). Overall, the NAD ${ }^{+}$-binding domain seems to be the most mobile region due to the highest RMSD $(\sim 0.25 \mathrm{~nm})$ (Fig. 2C). However, parts of these domains showed different degrees of structural fluctuations. Residues $152-262$ in $\mathrm{NAD}^{+}$-binding domain displays less mobility than the other part in the same domain (Fig. 2C). Residues 480-486 in oligomerization domain also shows low mobility than the other region in the same domain due to the short length (only 6 amino acids in this region). The rest of the protein showed low protein flexibility (RMSDs of $\sim 0.2 \mathrm{~nm}$ in Fig. $2 \mathrm{~B}-\mathrm{D}$ )

To explore protein dynamics, Principal Component Analysis (PCA) was performed on C $\alpha$ atoms. This revealed that the first eigenvector (principal component 1 (PC1)) accounts for the major motions and there are indications that the second eigenvector (principal component 2 (PC2)) could also play a role (Fig. S3). Overall, the direction of main motions obtained from PC1 and PC2 are similar for both simulations (Fig. S4). The scissor-like motion is observed in all simulations (Figs 3C and S4). Mostly, both PC1 and PC2 give the same inward scissoring movement for both Sim 1 and Sim 2, except PC2 for Sim 2 where outward scissoring-like motion is observed (Fig. S4). Due to the sharp peak of RMSFs extracted from PCA, the NAD ${ }^{+}$-binding region (residues 50-100) adjacent to the oligomerization domain appeared to be flexible and contributed to the protein dynamics in both simulations (Fig. 3). This result agrees well with our RMSD calculation. It would appear that the high RMSDs and RMSFs observed indicate that $\mathrm{NAD}^{+}$-binding domain is the major contributor to protein motion. Besides this, 

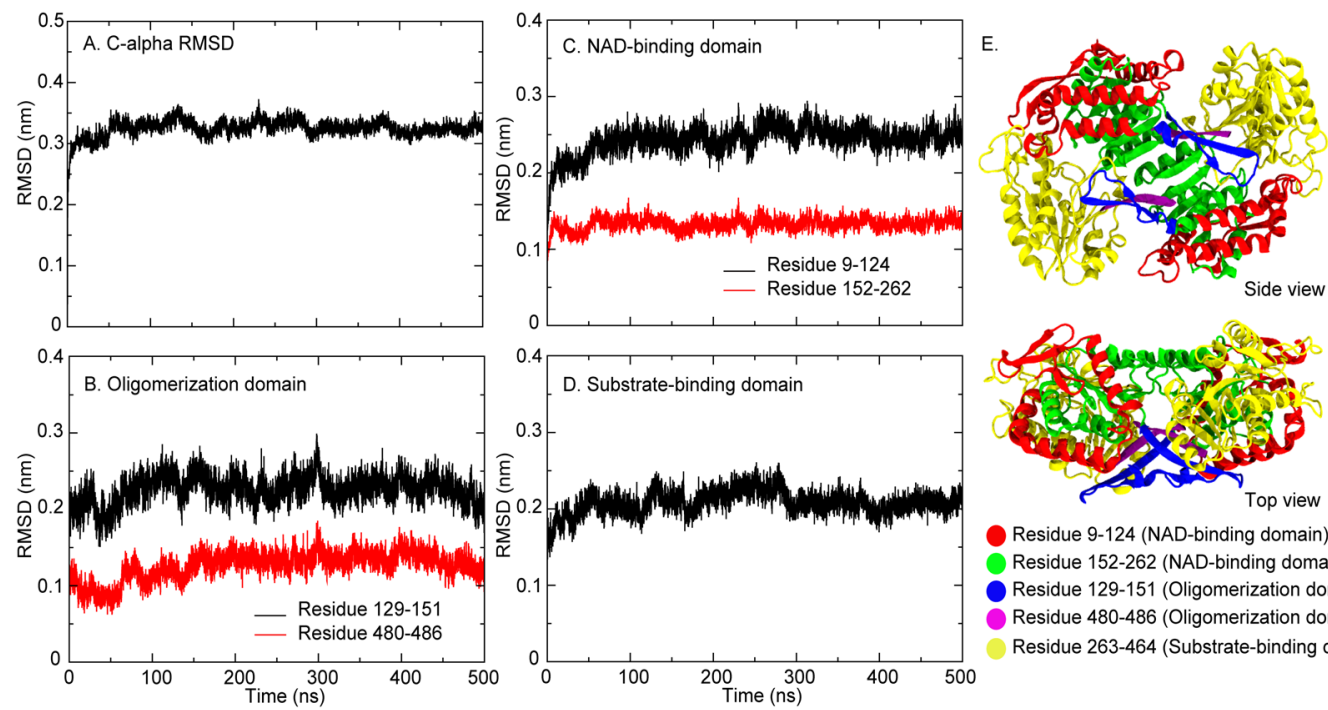

Residue 9-124 (NAD-binding domain) Residue 152-262 (NAD-binding domain) Residue 129-151 (Oligomerization domain) Residue 480-486 (Oligomerization domain) Residue 263-464 (Substrate-binding domain)

Figure 2. Average C $\alpha$ RMSD is shown in (A). Average RMSDs of each domain are displayed in (B,D). The cartoon views of each monomer (M1 and M2) are shown in (E). Colours have been used to demarcate the regions used for the calculation of RMSDs in (B) to (D).
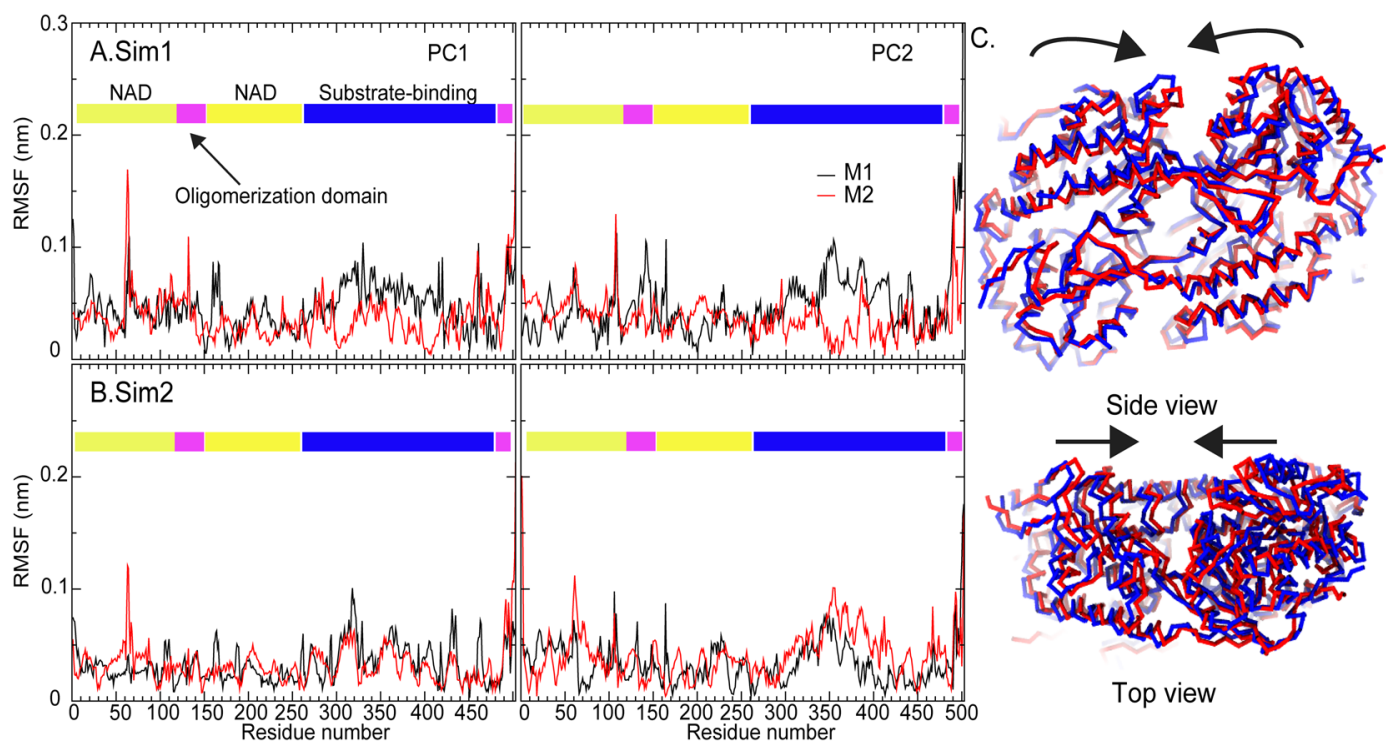

Top view

Figure 3. C $\alpha$ RMSFs computed from Principal Component Analysis (PCA) of (A) Sim 1 and (B) Sim 2. The structures on the right represent the major motions of $\mathrm{BADH} 2$ obtained from the principal component 1 (PC1) from Sim 1. The blue trace shows an initial conformation, while the red displays the final orientation. Arrows indicate the direction of protein motion.

the $\mathrm{C} \alpha$ atoms of the substrate-binding domains are also quite mobile (Fig. 3A,B). Furthermore, different degrees of backbone flexibilities observed from RMSFs reflect that dynamics of both monomers are not identical.

To evaluate protein-protein and protein-solvent interactions, average hydrogen bonds between monomer and solvent was calculated. Comparable monomer-water and inter-monomeric hydrogen bond interactions were observed in both simulations (Table 1). Fig. 4A summarizes the interactions between key amino acids inside the binding pocket. Hydrogen bonds between key residues (N162, Y163, L166, W170, E260, C294, C453, W459) and their adjacent residues were computed. The position of the interaction network can be divided into 3 groups and these are shown as coloured surfaces in Fig. 4B. We define a channel at the opposite side of the oligomerization region as the "front" part, whereas the "back" refers to the side in contact with the oligomerization domain (Fig. 4B). The first group, N162 and C294, previously reported as key to catalytic activity, are located on the front part of the substrate- and NAD ${ }^{+}$-binding sites (green and yellow surfaces in Fig. 4B). Both N162 and C294 form different degrees of hydrogen bonds. C294 seems to weakly hydrogen bond with adjacent amino acids $(<1 \%$ of hydrogen bonds with N162), whereas N162 forms a strong hydrogen bond with Q292 (>90\%). Apparently, C294 seems to be flexible, whilst N162 is tethered by Q292 inside a pocket. The blue surface in Fig. 4B represents the 


\begin{tabular}{|c|c|c|c|c|}
\hline & \multicolumn{2}{|l|}{ Sim 1} & \multicolumn{2}{|l|}{ Sim 2} \\
\hline & M1 & M2 & M1 & M2 \\
\hline Water & $946.28 \pm 18.85$ & $952.27 \pm 18.96$ & $955.13 \pm 5.18 .05$ & $954.92 \pm 18.42$ \\
\hline M1:M2 & \multicolumn{2}{|l|}{$42.21 \pm 5.11$} & \multicolumn{2}{|l|}{$37.83 \pm 5.68$} \\
\hline
\end{tabular}

Table 1. Average number of hydrogen bonds observed throughout each simulation. M1:M2 indicates the number of hydrogen bonds occurring between monomer 1 (M1) and monomer 2 (M2).
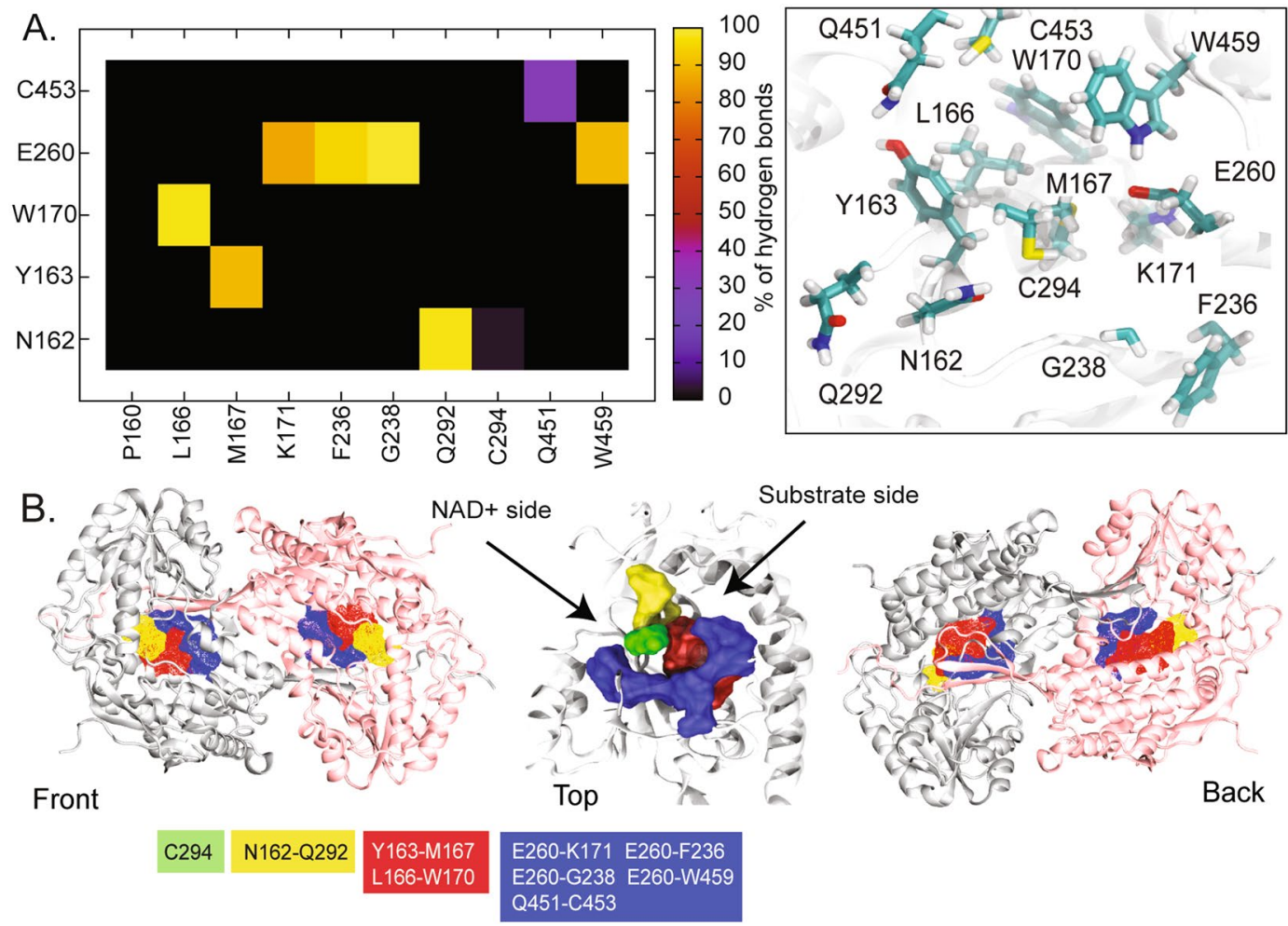

Figure 4. (A) The percentage of hydrogen bonds occurring throughout all simulations in the regions of substrate- and $\mathrm{NAD}^{+}$-binding domains. (B) Key amino acids inside the pocket are also shown in licorice format on the right. (B) Front and back views of dimeric BADH2 with red, yellow, and blue surfaces representing the location of each interaction network. The interactions in these regions are shown below in coloured boxes.

second group (E260, C453, and 459) and the red one stands for the third group at the base (Y163, L166, W170) where strong hydrogen bonds between Y163-M167 and L166-W170 were observed (Fig. 4A). Additionally, one of the key catalytic residues, E260, located at the side of the binding sites formed a strong hydrogen-bonding network with many nearby residues. K171, F236, G238, and W459 were observed to form long lasting hydrogen bonds with E260 (>80\% in Fig. 4A). Our results agree with a study that showed that E260 strongly interacts with W459, but BADH2 does not form any significant E260-E470 interaction as found in AMADH ${ }^{10}$. Besides this, a transient interaction between C453-Q451 was also captured. To date, both computational and experimental studies of ALDH family ${ }^{10,20-23}$ suggest the binding site of a substrate is at the back of protein, whereas the front area accommodates $\mathrm{NAD}^{+}$binding. $\mathrm{NAD}^{+}$was predicted to enter the binding pocket from the front and a substrate goes to the opposite opening (Figs 4B and 5A) ${ }^{21}$. The catalytic C294 is located in the middle of a cavity (green surface in Fig. 4B (center)). Residues Y163, L166, M167, and W170 are aligned at the base of the substrate-binding site (red surface in Fig. 4B (center)), whilst the N162-Q292 interaction drags the N162's sidechain towards the $\mathrm{NAD}^{+}$binding site. Upon substrate binding, C294 and E260 were reported to be involved in the formation of key hemithioacetal-enzyme intermediate in assistance with $\mathrm{NAD}^{+}$. The nucleophile $\mathrm{C} 294$ attacks an aldehyde carbonyl of the bound substrate and the deprotonation of a substrate is undertaken by E260. Unlike catalytic C294 and E260, N162 appears to play a minor role in stabilizing an intermediate by forming an oxyanion hole ${ }^{24}$. Our simulations demonstrate the high flexibility of C294 which supports the role of the nucleophile C294's ability to attack a free carbonyl group of a bound substrate. On the contrary, N162 and E260 appear to be rigid due to strong interactions with their neighbours. Such interactions tethering N162 and E260 may help to shape a suitable environment for a catalytic activity.

It has been reported that in ALDH, electrostatic attractions serve as a major player for dimeric association ${ }^{25}$. In the oligomerization domain, there is an accumulation of charged residues at the base and some are distributed along the latching area (Fig. 5A). In the latching region, side chains of charged residues appear to point towards 
A.

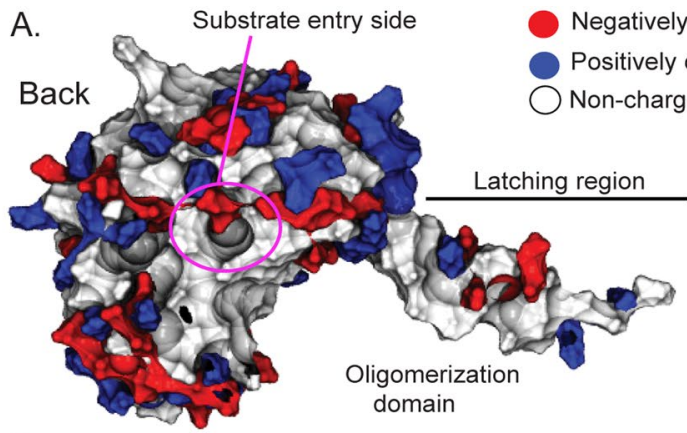

B.

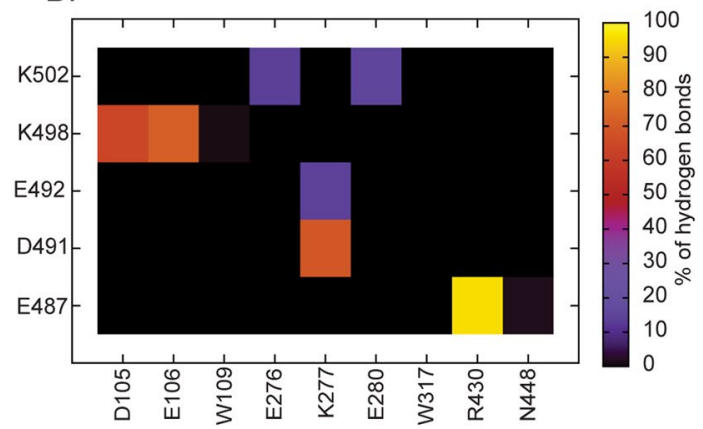

Negatively charged residues

Positively charged residues

Non-charged residues

Figure 5. (A) Front and back surface views of BADH2 monomer. (B) The percentage of hydrogen bonds occurring throughout the simulations at the dimeric interface. (C) Dimeric BADH2 with key residues at the dimer interface.

the bulk, while non-charged amino acids seem to line the dimer-dimer interface (Fig. 5A). Here, key electrostatic interactions on the latching region have been identified for the first time. Figure 5B,C illustrates key inter-subunit salt bridges between dimers and their locations. Charged residues at the $\mathrm{C}$-terminus seem to play a role in dimer assembly. Permanent R430-E487 hydrogen bond (>90\%) highlights its key role in dimer association (Fig. 5B). Moreover, not only moderate hydrogen bonds of K498-D105, K498-E106, D491-K277 ( 50-60\%), but also transient interactions of K277-E492, K502-E276, and K502-E280 (<40\%) have been captured (Fig. 5B,C). Despite the fact that uncharged dimer contacts have been observed, inter-subunit salt bridges have also been observed between charged residues (E487, D491, E492, K498, and K502) on the latching loop between adjacent monomers. These act as clips that hold the dimer together.

\section{Discussion}

In this study, the dynamic properties of dimeric $\mathrm{BADH} 2$ were investigated. The $\mathrm{NAD}^{+}$-binding domain was found to be very mobile and contributes to most of the dynamics of BADH2. Key interactions in the ligand binding pocket have also been revealed. N162 and E260 form strong hydrogen bonds with nearby residues resulting in a rigid conformation. The strong interaction network may help to shape a suitable cavity size and dimension that fits both substrate and cofactor. In contrast, C294 was found to be very mobile inside the pocket. It weakly interacted with N162. Its flexibility could facilitate the attack of carbonyl on an aldehyde substrate. The mobility of the catalytic cysteine is similar to that of other ALDHs from previous MD and QM/M studies ${ }^{22,23,26}$. Furthermore, key inter-subunit salt bridges contributing to dimerization have been identified for the first time. Although the dimer interface is lined by uncharged residues, both electronegative and electropositive residues on the latching region - E487, D491, E492, K498, and K502 - have been found to form strong interactions with charged residues on the other monomer. Such charged residues seem to play a role in dimer formation and stability.

Within the ALDH family, research interest has been drawn to protein-ligand binding affinities and catalytic mechanisms ${ }^{16,22,23,26,27}$. The highly conserved sequence in the catalytic pocket defines similar residues for catalysis. The overall picture of the reaction mechanism seems to be well-defined. However, not many studies have been devoted to protein structure and dynamics. Our work appears to be the first to address this gap. Each ALDH protein must have a clearly differentiated mechanism so as to obtain its unique function. Thus, understanding the ligand-protein interactions, dynamics, and detailed enzymatic mechanism of each protein remain crucial. An insight into the structure and function of $\mathrm{BADH} 2$ will be useful for future improvement of rice fragrance, which could lead to an enhancement of rice quality and market price.

\section{Methods}

The sequence of BADH2 was obtained from Uniprot (Entry: O24174). A BLAST search based on the protein data bank revealed that the structures most similar to BADH2 was the 3-dimensional structure of aminoaldehyde dehydrogenase (AMADH) from Pisum sativum (PDB codes: 3IWK and 3IWJ) with a sequence similarity of $76.54 \%$. The 3D model of dimeric BADH2 (503 amino acids each) was built by homology modelling using MODELLER (version 9.17) ${ }^{28}$. The quality of the model was checked by Ramachandran structure validation 
using RAMPAGE (http://mordred.bioc.cam.ac.uk/ rapper/rampage.php) ${ }^{29}$. The model developed had residues in favored (96.6\%), allowed (2.8\%) and outlier (0.6\%) regions (Fig. S1 in Supplementary Information (SI)). Structures with residues over $90 \%$ in the favored region display a good structure quality as reported in both computational and experimental studies ${ }^{30-33}$. In this work, M1 refers to monomer 1 and M2 is for monomer 2 . The protonation states of charged amino acids were set as that at physiological $\mathrm{pH}$.

We employed GROMACS 5.0 package (www.gromacs.org) ${ }^{34}$ with Amberff99SB-ILDN force field. Particle mesh Ewald (PME) ${ }^{35}$ method with a Fourier spacing of $0.12 \mathrm{~nm}$ and a short range cut-off of $1 \mathrm{~nm}$ was used for electrostatic treatment. The system contained dimeric BADH2 and 86,416 TIP3P water molecules. Counter ions were added to neutralize the simulation system. Energy minimization of 1000 steps was performed to remove bad contacts using steepest descent algorithm followed by $10 \mathrm{~ns}$ of equilibration where the protein atoms were restrained with a force constant of $1000 \mathrm{~kJ} \mathrm{~mol}^{-1} \mathrm{~nm}^{-2}$. Next, two $0.5 \mu$ s production runs were performed. The simulations were conducted in the constant number of particles, pressure, and temperature (NPT) ensemble. The Berendsen algorithm at 1 bar with a coupling constant $\tau_{p}=1 \mathrm{ps}$ was used for pressure coupling. The temperature of the protein and solution were coupled separately using the v-rescale thermostat ${ }^{36}$ at $300 \mathrm{~K}$ with a coupling constant $\tau_{\mathrm{t}}=0.1 \mathrm{ps}$. The time step of $2 \mathrm{fs}$ was used for integration. The coordinates were recorded every $2 \mathrm{ps}$.

Simulations trajectories were analyzed using GROMACS tools and in-house code. RMSD and RMSF calculations were performed using the initial structure from each of the two simulations. The hydrogen bonds were computed using g_hbond with default parameters (The hydrogen-donor-acceptor cutoff angle is $30^{\circ}$ and the cutoff radius (X-acceptor) is $0.35 \mathrm{~nm}$ ). VMD was used for visualization and generation of images ${ }^{37}$.

\section{References}

1. Bhattacharjee, P., Singhal, R. S. \& Kulkarni, P. R. Basmati rice: a review. Int J Food Sci Tech 37, 1-12, https://doi.org/10.1046/j.13652621.2002.00541.x (2002).

2. Garg, A. K. et al. Light-regulated overexpression of an Arabidopsis phytochrome A gene in rice alters plant architecture and increases grain yield. Planta 223, 627-636, https://doi.org/10.1007/s00425-005-0101-3 (2006).

3. Buttery, R. G., Ling, L. C., Juliano, B. O. \& Turnbaugh, J. G. Cooked rice aromac and 2-acetyl-1-pyrroline. Journal of agricultural and food chemistry 31, 823-826 (1983).

4. Bradbury, L. M., Fitzgerald, T. L., Henry, R. J., Jin, Q. \& Waters, D. L. The gene for fragrance in rice. Plant biotechnology journal 3, 363-370, https://doi.org/10.1111/j.1467-7652.2005.00131.x (2005).

5. Fitzgerald, T. L., Waters, D. L. \& Henry, R. J. Betaine aldehyde dehydrogenase in plants. Plant biology 11, 119-130, https://doi. org/10.1111/j.1438-8677.2008.00161.x (2009).

6. Liu, Z. J. et al. The first structure of an aldehyde dehydrogenase reveals novel interactions between NAD and the Rossmann fold. Nat Struct Biol 4, 317-326 (1997).

7. Mitsuya, S., Yokota, Y., Fujiwara, T., Mori, N. \& Takabe, T. OsBADH1 is possibly involved in acetaldehyde oxidation in rice plant peroxisomes. Febs Lett 583, 3625-3629, https://doi.org/10.1016/j.febslet.2009.10.039 (2009).

8. Bradbury, L. M., Gillies, S. A., Brushett, D. J., Waters, D. L. \& Henry, R. J. Inactivation of an aminoaldehyde dehydrogenase is responsible for fragrance in rice. Plant molecular biology 68, 439-449, https://doi.org/10.1007/s11103-008-9381-x (2008).

9. Fujiwara, T. et al. Enzymatic characterization of peroxisomal and cytosolic betaine aldehyde dehydrogenases in barley. Physiol Plant 134, 22-30, https://doi.org/10.1111/j.1399-3054.2008.01122.x (2008).

10. Tylichova, M. et al. Structural and Functional Characterization of Plant Aminoaldehyde Dehydrogenase from Pisum sativum with a Broad Specificity for Natural and Synthetic Aminoaldehydes. J Mol Biol 396, 870-882, https://doi.org/10.1016/j.jmb.2009.12.015 (2010).

11. Chen, S. et al. Badh2, encoding betaine aldehyde dehydrogenase, inhibits the biosynthesis of 2-acetyl-1-pyrroline, a major component in rice fragrance. The Plant cell 20, 1850-1861, https://doi.org/10.1105/tpc.108.058917 (2008).

12. Niu, X. L. et al. RNAi-directed downregulation of OsBADH2 results in aroma (2-acetyl-1-pyrroline) production in rice (Oryza sativa L.). Bmc Plant Biol 8, https://doi.org/10.1186/1471-2229-8-100 (2008).

13. Bourgis, F. et al. Characterization of the major fragance gene from an aromatic japonica rice and analysis of its diversity in Asian cultivated rice. TAG. Theoretical and applied genetics. Theoretische und angewandte. Genetik 117, 353-368, https://doi.org/10.1007/ s00122-008-0780-9 (2008).

14. Shi, W. W., Yang, Y., Chen, S. H. \& Xu, M. L. Discovery of a new fragrance allele and the development of functional markers for the breeding of fragrant rice varieties. Mol Breeding 22, 185-192, https://doi.org/10.1007/s11032-008-9165-7 (2008).

15. Kovach, M. J., Calingacion, M. N., Fitzgerald, M. A. \& McCouch, S. R. The origin and evolution of fragrance in rice (Oryza sativa L.). Proceedings of the National Academy of Sciences of the United States of America 106, 14444-14449, https://doi.org/10.1073/ pnas.0904077106 (2009).

16. Wongpanya, R. et al. Biochemical and enzymatic study of rice BADH wild-type and mutants: an insight into fragrance in rice. The protein journal 30, 529-538, https://doi.org/10.1007/s10930-011-9358-5 (2011)

17. Jiamsomboon, K., Treesuwan, W. \& Boonyalai, N. Dissecting substrate specificity of two rice BADH isoforms: Enzyme kinetics, docking and molecular dynamics simulation studies. Biochimie 94, 1773-1783, https://doi.org/10.1016/j.biochi.2012.04.009 (2012).

18. Kamaraj, B. \& Purohit, R. In-silico analysis of Betaine Aldehyde Dehydrogenase 2 of Oryza sativa and significant mutations responsible for fragrance. J Plant Interact 8, 321-333, https://doi.org/10.1080/17429145.2012.758785 (2013).

19. Thompson, J. D., Higgins, D. G. \& Gibson, T. J. Clustal-W - Improving the Sensitivity of Progressive Multiple Sequence Alignment through Sequence Weighting, Position-Specific Gap Penalties and Weight Matrix Choice. Nucleic Acids Research 22, 4673-4680, https://doi.org/10.1093/nar/22.22.4673 (1994).

20. Johansson, K. et al. Structure of betaine aldehyde dehydrogenase at 2.1 A resolution. Protein Sci 7, 2106-2117, https://doi. org/10.1002/pro.5560071007 (1998).

21. Halavaty, A. S. et al. Structural and functional analysis of betaine aldehyde dehydrogenase from Staphylococcus aureus. Acta crystallographica. Section D, Biological crystallography 71, 1159-1175, https://doi.org/10.1107/S1399004715004228 (2015).

22. Wymore, T., Nicholas, H. B. \& Hempel, J. Molecular dynamics simulation of class 3 aldehyde dehydrogenase. Chem-Biol Interact 130, 201-207, https://doi.org/10.1016/S0009-2797(00)00278-7 (2001).

23. Wymore, T. et al. Molecular recognition of aldehydes by aldehyde dehydrogenase and mechanism of nucleophile activation. Proteins-Structure Function and Bioinformatics 57, 758-771, https://doi.org/10.1002/prot.20256 (2004).

24. Luo, M. \& Tanner, J. J. Structural Basis of Substrate Recognition by Aldehyde Dehydrogenase 7A1. Biochemistry 54, 5513-5522, https://doi.org/10.1021/acs.biochem.5b00754 (2015).

25. Rodriguez-Zavala, J. S. \& Weiner, H. Structural aspects of aldehyde dehydrogenase that influence dimer-tetramer formation. Biochemistry 41, 8229-8237, https://doi.org/10.1021/bi012081x (2002).

26. Wymore, T., Deerfield, D. W., Field, M. J., Hempel, J. \& Nicholas, H. B. Initial catalytic events in class 3 aldehyde dehydrogenase: MM and QM/MM simulations. Chem-Biol Interact 143, 75-84, https://doi.org/10.1016/S0009-2797(02)00175-8 (2003). 
27. Izaguirre, G., Pietruszko, R., Cho, S. \& MacKerrell, A. D. Human aldehyde dehydrogenase catalytic activity and structural interactions with coenzyme analogs. Journal of biomolecular structure \& dynamics 19, 429-447, https://doi.org/10.1080/07391102.2 $001.10506752(2001)$.

28. Eswar, N. et al. Comparative protein structure modeling using Modeller. Curr Protoc Bioinformatics Chapter 5, Unit5 6, https://doi. org/10.1002/0471250953.bi0506s15 (2006).

29. Lovell, S. C. et al. Structure validation by Calpha geometry: phi,psi and Cbeta deviation. Proteins 50, 437-450, https://doi. org/10.1002/prot.10286 (2003).

30. Bertinia, I., Cavallaro, G., Luchinat, C. \& Poli, I. A use of Ramachandran potentials in protein solution structure determinations. J Biomol Nmr 26, 355-366, https://doi.org/10.1023/A:1024092421649 (2003).

31. Avbelj, F. \& Baldwin, R. L. Role of backbone solvation and electrostatics in generating preferred peptide backbone conformations: Distributions of phi. Proceedings of the National Academy of Sciences of the United States of America 100, 5742-5747, https://doi. org/10.1073/pnas.1031522100 (2003).

32. Shamriz, S. \& Ofoghi, H. Design, structure prediction and molecular dynamics simulation of a fusion construct containing malaria pre-erythrocytic vaccine candidate, PfCelTOS, and human interleukin 2 as adjuvant. BMC bioinformatics 17, https://doi. org/10.1186/s12859-016-0918-8 (2016).

33. Honarparvar, B. \& Skelton, A. A. Molecular dynamics simulation and conformational analysis of some catalytically active peptides. J Mol Model 21, https://doi.org/10.1007/s00894-015-2645 (2015).

34. Lindahl, E., Hess, B. \& van der Spoel, D. GROMACS 3.0: a package for molecular simulation and trajectory analysis. Journal of Molecular modeling 7, 306-317 (2001).

35. Darden, T., York, D. \& Pedersen, L. Particle mesh Ewald: An N.log(N) method for Ewald sums in large systems. J. Chem. Phys. 98, 10089-10092 (1993).

36. Berendsen, H. J. C., Postma, J. P. M., van Gunsteren, W. F., DiNola, A. \& Haak, J. R. Molecular dynamics with coupling to an external bath. J. Chem. Phys. 81, 3684-3690 (1984).

37. Humphrey, W., Dalke, A. \& Schulten, K. VMD - Visual Molecular Dynamics. J. Mol. Graph. 14, 33-38 (1996).

\section{Acknowledgements}

P.P. would like to thank Kasetsart University Research and Development Institute (KURDI) (PY(D)156.60) for financial support. R.V. would like to thank United Arab Emirates University for a startup grant (Grant Number: G00001609).

\section{Author Contributions}

P.P. conceived the experiments, A.B., R.V. and P.P. conducted the experiments and A.B. and P.P. analysed the results. P.P. and R.V. wrote and reviewed the manuscripts.

\section{Additional Information}

Supplementary information accompanies this paper at https://doi.org/10.1038/s41598-018-31204-z.

Competing Interests: The authors declare no competing interests.

Publisher's note: Springer Nature remains neutral with regard to jurisdictional claims in published maps and institutional affiliations.

(c) (i) Open Access This article is licensed under a Creative Commons Attribution 4.0 International

License, which permits use, sharing, adaptation, distribution and reproduction in any medium or format, as long as you give appropriate credit to the original author(s) and the source, provide a link to the Creative Commons license, and indicate if changes were made. The images or other third party material in this article are included in the article's Creative Commons license, unless indicated otherwise in a credit line to the material. If material is not included in the article's Creative Commons license and your intended use is not permitted by statutory regulation or exceeds the permitted use, you will need to obtain permission directly from the copyright holder. To view a copy of this license, visit http://creativecommons.org/licenses/by/4.0/.

(c) The Author(s) 2018 